\title{
Order and Metric in the Stream Semantics of Elemental Concurrency
}

\author{
J.W. de Bakker ${ }^{1}$ and J.-J.Ch. Meyer ${ }^{2}$ \\ ${ }^{1}$ Centre for Mathematics and Computer Science, P.O. Box 4079, NL-1009 AB Amsterdam, \\ The Netherlands \\ 2 Department of Mathematics and Computer Science, Free University of Amsterdam, \\ The Netherlands
}

\begin{abstract}
Summary. Two denotational semantics for a language with simple concurrency are presented. The language has parallel composition in the form of the shuffle operation, in addition to the usual sequential concepts including full recursion. Two linear time models, both involving sets of finite and infinite streams, are given. The first model is order-theoretic and based on the Smyth order. The second model employs complete metric spaces. Various technical results are obtained relating the order-theoretic and metric notions. The paper culminates in the proof that the two semantics for the language considered coincide. The paper completes previous investigations of the same language, establishing the equivalence of altogether four semantic models for it.
\end{abstract}

\section{Introduction}

We present two denotational semantics for a language with simple concurrency, and prove their equivalence. The first semantics has an order-theoretic, the second a metric structure as underlying model. In the course of proving the equivalence theorem, a number of results are obtained relating the two structures which may be of some independent interest.

The first model will be based on the so-called Smyth order between sets of streams (in the sense of, e.g., $[10,11]$ ). This model was first developed in $[18,19]$. The second model introduces a distance between streams. In this way, the set of all streams is turned into a complete metric space, and familiar tools such as Banach's fixed point theorem become available. The metric model was first presented in [2]; essential inspiration for it was provided by [21].

Both models are of what has been called the 'linear time' variety. They are built on (sets of) sequences rather than on tree (-like) objects. For an overview of situations where the latter - also called 'branching time' - approach is preferable or even necessary, we refer to [3]. Briefly, once notions such as deadlock or global nondeterminacy are covered, branching time models or variations 
along the lines of ready or failure sets (see [22] for a systematic treatment) are required.

In the present paper we restrict ourselves to a very simple setting. The language $\mathscr{L}$ which we investigate has the familiar sequential notions (elementary or atomic actions, sequential composition), and in addition recursion, nondeterministic choice and parallel composition specifying the interleaving or merge of (sequences of) elementary actions. No forms of synchronization or communication are included: $\mathscr{L}$ is, indeed quite elementary. The motivation for its study is primarily that we are able to obtain an exhaustive analysis of its various semantic models - more about this in a moment -, rather than its intrinsic semantic interest. Still, we believe that the notions of recursion and merge are both fundamental in (the nature of) parallel computation, justifying our terminology of elemental concurrency.

Our paper may in fact be seen as the third in a series, completing the comparison of altogether four semantic models, viz. one operational, one metric denotational and two order-theoretic denotational semantics. The precise picture is the following:

1. In $[6,7]$ we have developed an operational $(\mathcal{O})$ and a metric denotational $(\mathscr{M})$ model (the same one has the one described below), and proved their equivalence. The operational semantics uses the transition systems of Hennessy and Plotkin $[15,23]$; as we saw already, the metric model goes back to [2].

2. In $[18,19]$ the Smyth order-theoretic semantics $\mathscr{S}$ for $\mathscr{L}$ was first proposed. A second order-theoretic semantics, $\mathscr{F}$, building upon ideas in [22], was designed by Olderog, see $[4,5]$ for details. This model uses sets of finite so-called observations rather than sets of possibly infinite streams; as order between the sets simple (reverse) set inclusion is used. In $[4,5]$ it was proved that the two order-theoretic structures - subject to certain conditions specification of which we omit here - are isomorphic. As an easy consequence, we obtain that $\mathscr{S}=\mathscr{F}$. (Roughly; the precise statement involves the isomorphism between the two structures.)

3. Altogether, we have four semantics for $\mathscr{L}$, viz. $\mathcal{O}, \mathscr{M}, \mathscr{S}$ and $\mathscr{F}$, and we know that $\mathcal{O}=\mathscr{M}$ and $\mathscr{S}=\mathscr{F}$. There remains the natural question whether $\mathscr{M}=\mathscr{S}$, and our paper answers this question affirmatively, thus completing (this branch of) the comparative semantics for elemental concurrency.

4. As a side remark pertaining to the relationship with branching time models, we recall that in [2] we also designed a branching time model for $\mathscr{L}$ (in terms of the processes as in [8]). Calling this semantics $\mathscr{B}$, we showed that, by applying the trace operation to $\mathscr{B}$ - collecting all paths in the tree-like object resulting from application of $\mathscr{B}$ to a statement -, we obtain $\mathscr{M}$. Thus, we proved that $\mathscr{M}=$ trace $\circ \mathscr{B}$.

Section 2 contains a few mathematical preliminaries, covering elementary definitions for metric spaces and complete partially ordered sets (cpo's). This section is almost as in [3]. Section 3 develops various basic semantic definitions: We define the set of streams as a cpo and as a metric space and similarly for the power set of the set of streams. Moreover, we define, for sets of streams (satisfying certain restrictions) the semantic operators of sequential composition, union and merge. The section culminates in the definitions of $\mathscr{S}$ and $\mathscr{M}$. As 
such, it may be seen as a tutorial introduction to previous work of the authors presenting these two models. In Sect. 4 we prove a number of technical results concerning the order-theoretic and metric structures, and their mutual relationship. Maybe the most important fact is the following: Let $\left(X_{i}\right)_{i}$ be a Smythordered chain of sets of streams (satisfying certain conditions). Then $\left(X_{i}\right)_{i}$ is also a Cauchy sequence in an appropriate metric space, and the order-theoretic and topological limits coincide. In the proof of this the compactness of the spaces concerned - a direct consequence of the finiteness of the alphabet of elementary actions - is employed. In Sect. 5 we establish the main result of the paper, viz. that $\mathscr{M}=\mathscr{S}$. The proof uses the properties relating metric and order obtained in Sect. 4. In addition, a proof technique closely resembling a method used in [7] (in Theorem 2.4.1 of that paper) is applied.

\section{Mathematical Preliminaries}

In this section we collect some basic definitions and properties concerning (i) metric spaces and (ii) complete partially ordered sets. Both structures will play a role in the denotational models to be presented in Sect. 3 and analyzed in Sect. 4 and 5.

\subsection{Elementary Definitions}

Let $X$ be any set. $\mathfrak{P}(X)$ denotes the powerset of $X$, i.e., the set of all subsets of $X . \mathfrak{P}_{\ldots}(X)$ denotes the set of all subsets of $X$ which have property $\ldots$. A sequence $x_{0}, x_{1}, \ldots$ of elements of $X$ is usually denoted by $\left(x_{i}\right)_{i=0}^{\infty}$ or, briefly $\left(x_{i}\right)_{i}$. Often, we shall have occasion to use the limit, supremum (sup), least upper bound (lub), etc, of a sequence $\left(x_{i}\right)_{i}$. We then use the notations $\lim x_{i}$, or, briefly, $\lim x_{i}, \sup x_{i}, \operatorname{lub} x_{i}$, etc. The notation $f: X \rightarrow Y$ expresses that $f$ is a function with domain $X$ and range $Y$. If $X=Y$ and, for $x \in X, f(x)=x$, we call $x$ a fixed point of $f$. We use $\mathbb{N}$ to denote the set of nonnegative integers.

\subsection{Metric Spaces}

Definition 2.1. A metric space is a pair $(M, d)$ with $M$ a set and $d$ (for distance) a mapping $d: M \times M \rightarrow[0,1]$ which satisfies the following properties:

a) $d(x, y)=0$ iff $x=y$,

b) $d(x, y)=d(y, x)$,

c) $d(x, y) \leqq d(x, z)+d(z, y)$.

If clause a) is replaced by the weaker $\left.\mathrm{a}^{\prime}\right): d(x, y)=0$ if $x=y$, we call $(M, d)$ a pseudometric space.

Definition 2.2. Let $(M, d)$ be a metric space. 
a) Let $\left(x_{i}\right)_{i}$ be a sequence in $M$. We say that $\left(x_{i}\right)_{i}$ converges to an element $x$ in $M$ called its limit, whenever we have:

$$
\forall \varepsilon>0 \quad \exists N \in \mathbb{N} \forall n>N\left[d\left(x, x_{n}\right)<\varepsilon\right] .
$$

A sequence $\left(x_{i}\right)_{i}$ in $M$ is a convergent sequence if it converges to $x$ for some $x \in X$.

b) A sequence $\left(x_{i}\right)_{i}$ is called a Cauchy sequence whenever we have

$$
\forall \varepsilon>0 \quad \exists N \in \mathbb{N} \forall n, m>N\left[d\left(x_{n}, x_{m}\right)<\varepsilon\right] .
$$

c) The space $(M, d)$ is called complete whenever each Cauchy sequence converges to an element in $M$.

d) A subset $X$ of a complete space $(M, d)$ is called closed whenever each Cauchy sequency in $X$ converges to an element of $X$.

Definition 2.3. a) Let $\left(M_{1}, d_{1}\right)$ and $\left(M_{2}, d_{2}\right)$ be two metric spaces. We call the spaces isometric if there exists a bijection $f: M_{1} \rightarrow M_{2}$ such that, for all $x, y \in M_{1}$, $d_{2}(f(x), f(y))=d_{1}(x, y)$.

b) Let $\left(M_{1}, d_{1}\right)$ and $\left(M_{2}, d_{2}\right)$ be two metric spaces. We call the function $f: M_{1} \rightarrow M_{2}$ continuous, whenever, for each sequence $\left(x_{i}\right)_{i}$ with limit $x$ in $M_{1}$, we have that $\lim _{i} f\left(x_{i}\right)=f(x)$.

c) Let $(M, d)$ be a metric space and $f: M \rightarrow M$. We call $f$ contracting if there exists a real constant $c, 0 \leqq c<1$, such that, for all $x, y \in M, d(f(x), f(y)) \leqq c$. $d(x, y)$.

Proposition 2.4. a) Each contracting function is continuous.

b) (Banach's fixed point theorem). Let $(M, d)$ be complete and $f: M \rightarrow M$ contracting. Then $f$ has a unique fixed point, which can be obtained as the limit of the (Cauchy) sequence $x_{0}, f\left(x_{0}\right), f\left(f\left(x_{0}\right)\right), \ldots$ for arbitrary $x_{0}$.

For each metric space $(M, d)$ it is possible to define a complete metric space $(\tilde{M}, \tilde{d})$ such that $(M, d)$ is isometric to a (dense) subspace of $(\tilde{M}, \tilde{d})$. In fact, we may take for $(\tilde{M}, \widetilde{d})$ the pseudo-metric space of all Cauchy sequences $\left(x_{i}\right)_{i}$ in $M$ with distance $d\left(\left(x_{i}\right)_{i},\left(y_{i}\right)_{i}\right)=\lim _{i} d\left(x_{i}, y_{i}\right)$ which is turned into a metric space

by taking equivalence classes with respect to the equivalence relation $\left(x_{i}\right)_{i} \equiv\left(y_{i}\right)_{i}$ iff $d\left(\left(x_{i}\right)_{i},\left(y_{i}\right)_{i}\right)=0 . M$ is embedded into $\tilde{M}$ by identifying each $x \in M$ with the constant Cauchy sequence $\left(x_{i}\right)_{i}$ with $x_{i}=x, i=0,1, \ldots$ in $\tilde{M}$.

For each metric space $(M, d)$ we can define a metric $\tilde{d}$ on the collection of its nonempty closed subsets, denoted by $\mathfrak{P}_{n c}(M)$, as follows:

Definition 2.5 (Hausdorff distance $\widetilde{d}$ ). Let $(M, d)$ be a metric space, and let $X$, $Y$ be nonempty subsets of $M$. We put

a) $d^{\prime}(x, Y)=\inf _{y \in Y} d(x, y)$.

b) $\widetilde{d}(X, Y)=\max \left(\sup _{x \in X} d^{\prime}(x, Y), \sup _{y \in Y} d^{\prime}(y, X)\right)$. 
We have the following theorem which is quite useful in our metric denotational models:

Proposition 2.6. Let $(M, d)$ be a metric space and $\tilde{d}$ as in Definition 2.5 .

a) $\left(\mathfrak{P}_{n c}(M), \mathcal{d}\right)$ is a metric space.

b) If $(M, d)$ is complete then $\left(\mathfrak{P}_{n c}(M), \widetilde{d}\right)$ is complete. Moreover, for $\left(X_{i}\right)_{i}$ a Cauchy sequence in $\left(\mathfrak{P}_{n c}(M), \mathcal{d}\right)$ we have

$$
\lim _{i} X_{i}=\left\{\lim _{i} X_{i}: x_{i} \in X_{i},\left(x_{i}\right)_{i} \text { a Cauchy sequence in } M\right\} .
$$

Proofs of Proposition 2.6 can be found e.g. in [12] or [13]. The proposition is due to Hahn [14]; the proof is also repeated in [8]. We close this subsection with a few definitions and properties relating to compact spaces and sets. First some terminology. A subset $X$ of a space $(M, d)$ is open if its complement $M \backslash X$ is closed. An (open) cover of a set $X$ is a family of (open) sets $Y_{i}, i \in I$, such that $X \subseteq \bigcup_{i \in I} Y_{i}$.

Definition 2.7. Let $(M, d)$ be a metric space.

a) $(M, d)$ is called compact whenever each open cover of $M$ has a finite subcover.

b) A subset $X$ of $M$ is called compact whenever each open cover of $X$ has a finite subcover.

Proposition 2.8. a) Each closed subset of a compact space is compact.

b) If $X$ is compact and $f$ is continuous then $f(X)$ is compact.

c) $X$ is compact iff there is a Cauchy sequence $\left(X_{i}\right)_{i}$ (with respect to the metric of Definition 2.5) of finite sets such that $X=\lim X_{i}$.

d) $(M, d)$ is compact whenever each infinite sequence $\left(x_{i}\right)_{i}$ has a convergent subsequence.

e) A subset $X$ of a metric space $(M, d)$ is compact whenever each infinite sequence $\left(x_{i}\right)_{i}, x_{i} \in X$, has a subsequence converging to an element of $X$.

In the final definition and proposition of this subsection we suppress explicit mentioning of the metrics involved. For $f$ a function: $M_{1} \rightarrow M_{2}$ we define $\hat{f}$ : $\mathfrak{P}_{n c}\left(M_{1}\right) \rightarrow \mathfrak{P}_{n c}\left(M_{2}\right)$ by $\hat{f}(M)=\{f(x): x \in X\}$. We have the following result from Rounds ([24]):

Proposition 2.9. Let $f$ be a function from a compact metric space $M_{1}$ to a compact metric space $M_{2}$.

The following three statements are equivalent:

a) $f$ is continuous.

b) $\hat{f}: \mathfrak{P}_{n c}\left(M_{1}\right) \rightarrow \mathfrak{P}_{n c}\left(M_{2}\right)$ is continuous with respect to the Hausdorff metric $(s)$.

c) For $X \in \mathfrak{P}_{n c}\left(M_{1}\right), \hat{f}(X) \in \mathfrak{P}_{n c}\left(M_{2}\right)$ and, for $\left(X_{i}\right)_{i}$ a decreasing $\left(X_{i} \supseteq X_{i+1}\right.$, $i=0,1,2, \ldots)$ chain of elements in $\mathfrak{P}_{n c}\left(M_{1}\right)$ we have

$$
\hat{f}\left(\bigcap_{i} X_{i}\right)=\bigcap_{i} \hat{f}\left(X_{i}\right) .
$$




\subsection{Complete Partially Ordered Sets}

Definition 2.10. a) A partial order (po) is a pair $(C$, $\subseteq)$ where $C$ is a set and $\subseteq$ a relation on $C$ (subset of $C \times C$ ) satisfying

$1 x \sqsubseteq x$,

2 if $x \sqsubseteq y$ and $y \sqsubseteq x$ then $x=y$,

3 if $x \sqsubseteq y$ and $y \sqsubseteq z$ then $x \sqsubseteq z$.

If $\subseteq$ satisfies only 1 and 3 it is called a preorder.

b) An (ascending) chain in $C$, ) is a sequence $\left(x_{i}\right)_{i}$ such that $x_{i} \sqsubseteq x_{i+1}, i$ $=0,1, \ldots$. The chain is called infinitely often increasing of $x_{i} \neq x_{i+1}$ for infinitely many $i$.
c) For $X \leqq C$ we call $y \in C$ the least upperbound (lub) of $X$ if
$1 \forall x \in X[x \sqsubseteq y]$,
$2 \forall z \in C[\forall x \in X[x \sqsubseteq z] \Rightarrow y \sqsubseteq z]$.

Definition 2.11. A complete partially ordered set (cpo) is a triple $(C, \sqsubseteq, \perp)$ with $(C, \sqsubseteq)$ a po and $\perp \in C$ such that
a) $\forall x \in C[\perp \subseteq x]$.
b) Each chain $\left(x_{i}\right)_{i}$ in $C$ has a lub in $C$.

For "the cpo $(C, \subseteq, \perp)$ " we often simply write "the cpo $C$ ".

Definition 2.12 (continuity). Let $C_{1}$ and $C_{2}$ be cpo's.

a) A function $f: C_{1} \rightarrow C_{2}$ is called monotonic whenever for all $x_{1}, x_{2} \in C_{2}$, if $x_{1} \sqsubseteq x_{2}$ then $f\left(x_{1}\right) \subseteq f\left(x_{2}\right)$.

b) A function $f: C_{1} \rightarrow C_{2}$ is called continuous whenever it is monotonic and, for each chain $\left(x_{i}\right)_{i}$ in $C_{1}$ we have $f\left(\operatorname{lub}_{i} x_{i}\right)=\underset{i}{\operatorname{lub}} f\left(x_{i}\right)$.

Proposition 2.13. Let $f$ be a continuous mapping from a cpo $C$ into itself: $f$ has a least fixed point $\mu f$ satisfying

$1 f(\mu f)=\mu f$,
2 if $f(y) \subseteq y$ then $\mu f \sqsubseteq y$,
$3 \mu f=\underset{i}{i} f^{i}(\perp)$, where $f^{0}=\lambda x \cdot x, f^{i+1}=f \circ f^{i}$.

Definition 2.14. a) A subset $X$ is called flat whenever, for all $x, y \in X, x \sqsubseteq y$ implies $x=y$.

b) A subset $X$ of a cpo $C$ is called closed whenever, for each infinitely often increasing chain $\left(x_{i}\right)_{i}$ of elements in $C$ such that, for all $i=0,1, \ldots$ we have that $x_{i} \sqsubseteq y_{i}$ for some $y_{i} \in X$, it follows that lub $x_{i} \in X$.

This definition of closed appears in [1] and [17]. We now introduce a number of preorders on $\mathfrak{P}(C)$, for $(C, \subseteq, \perp)$ a cpo.

Definition 2.15. a) The Smyth preorder $\varsigma_{s}: X \sqsubseteq_{S} Y$ iff $\forall y \in Y \exists x \in X[x \sqsubseteq y]$.

b) The Hoare preorder $\subseteq_{H}: X \subseteq_{H} Y$ iff $\forall x \in X \exists y \in Y[x \sqsubseteq y]$. 
c) The Egli-Milner preorder $\sqsubseteq_{E M}: X \sqsubseteq_{E M} Y$ iff $X \sqsubseteq_{S} Y$ and $X \sqsubseteq_{H} Y$.

None of the three preorders is, a partial order. In fact, we may take the two sets $X=\{x, y, z\}$ and $Y=\{x, z\}$ with $x \sqsubseteq y$ and $y \sqsubseteq z$ as a counterexample. In subsequent sections, only $\varsigma_{s}$ will be used. The other preorders are included for completeness' sake.

\section{Stream Semantics for Elemental Concurrency}

We introduce a simple language $\mathscr{L}$ with concurrency and design two denotational semantics for it. The first semantic function is called $\mathscr{S}$ (for Smyth-like ordertheoretic) and the second $\mathscr{M}$ (for metric). In subsequent sections we shall develop the tools for proving the equivalence $\mathscr{S}=\mathscr{M}$.

We recall from the introduction that we already showed in previous papers:

(i) For $\mathscr{F}$ the denotational semantics based on the cpo of (sets of) finite observations, $\mathscr{F}=\mathscr{S}$ (modulo the isomorphism linking the two cpo's.

(ii) For $\mathcal{O}$ the operational semantics based on transition systems, $\mathcal{C}=\mathscr{U}$. (In addition, we know that

(iii) For $\mathscr{B}$ the (metric) branching time semantics, trace $\bullet \mathscr{B}=\mathscr{M}$.)

We start the section with a description of the syntax of $\mathscr{L}$. Elements of $\mathscr{L}$ will be called statements or, occasionally, processes, and we use $s, t$ to range over $\mathscr{L}$. The language $\mathscr{L}$ is what we like to call a uniform language: its elementary actions are left uninterpreted. No constructs such as (individual) variables, assignments or tests are present in the syntax, and neither do we employ notions such as states in the semantics. In fact, statements in $\mathscr{L}$ may well be seen as (pieces of) grammar which prescribe the generation of finite or infinite sequences of symbols (or actions), and our semantic studies may shed light on questions in formal language theory as well.

For the syntax of $\mathscr{L}$ we need two classes of terminal elements:

1. The class $A$, with typical elements $a, b, \ldots$, of elementary actions. For $A$ we take an arbitrary (but finite) alphabet. Finiteness of $A$ results in compactness of the spaces concerned; see below.

2. The class $\mathscr{P}^{2}$ ar, with typical elements $x, y, \ldots$, of process variables. For $\mathscr{P}_{v a}$ \& we take some infinite set of symbols: it is convenient to have an infinite supply of fresh process variables. Process variables play a role in the syntactic construct for recursion as we shall see in a moment.

We now give, in a self-explanatory notation,

Definition 3.1 (syntax for $\mathscr{L}$ ).

$$
s:=a\left|s_{1} ; s_{2}\right| s_{1} \cup s_{2}\left|s_{1} \| s_{2}\right| x \mid \mu x[s] .
$$

A statement $s$ is of one of the following six forms:

- an elementary action a

- the sequential composition $s_{1} ; s_{2}$ of statements $s_{1}$ and $s_{2}$

- the nondeterministic choice $s_{1} \cup s_{2}$ : it is executed by executing $s_{1}$ or $s_{2}$ chosen nondeterministically 
- the concurrent execution $s_{1} \| s_{2}$, modelled by arbitrarily interleaving the elementary actions of $s_{1}$ and $s_{2}$

- a process variable $x$ which is (normally) used in

- the recursive construct $\mu x[s]$ : its execution amounts to execution of $s$ where occurrences of $x$ in $s$ are executed by (recursively) executing $\mu x[s]$. For example, with the definitions to be proposed presently, the intended meaning of $\mu x[(a ; x) \cup b]$ is the set $a^{*} \cdot b \cup\left\{a^{\omega}\right\}$. (Here $a^{\omega}$ denotes the infinite sequence of a's.)

The prefix $\mu x \cdots$ binds occurrences of $x$ in ... in the usual way, inducing the familiar notions of free and bound (occurrences of) process variables. We shall call a statement closed if it has no free occurrences of process variables.

We continue with the development of the two semantic models. For both of them we need various basic definitions which we may use to build the structures in which our semantics are defined. Apart from an occasional point of presentation, no new material is presented here: the definitions stem originally from $[18,19]$ and [2], and are included also in papers such as [3-7].

We begin with the definition of the set of streams over $A$, denoted by $A^{\text {st }}$ (cf. e.g. $[10,11]$ ). Let $\perp$ be a symbol not in $A$.

Definition 3.2 (streams). $A^{\text {st }}=A^{*} \cup A^{*} \cdot\{\perp\} \cup A^{\omega}$.

Here $A^{*}\left(A^{\omega}\right)$ denotes the set of all finite (infinite) words over $A$. We use $\varepsilon$ to denote the empty sequence. $A^{*} \cdot\{\perp\}$ is the collection of all finite words over $A$, followed by the $\perp$-symbol. We use $u, v, w$ to range over $A^{\text {st }}$. We recall (from Sect. 2.1) the notation $\mathfrak{P} . .\left(A^{\text {st }}\right)$ for the collection of all subsets of $A^{\text {st }}$ with property .... Usually, we abbreviate $\mathfrak{P} . . .\left(A^{\text {st }}\right)$ to $\mathbf{S} \ldots$. We shall use $X, Y, Z$ to range over $\mathbf{S}$.

The first group of basic definitions is assembled in

Definition 3.3. a) The function strip: $A^{\text {st }} \rightarrow A^{*} \cup A^{\omega}$. We put $\operatorname{strip}(u)=u$ for $u \in A^{*} \cup A^{\omega}$, and $\operatorname{strip}(u)=u^{\prime}$ for $u=u^{\prime} \perp$, with $u^{\prime} \in A^{*}$.

b) The prefix order $\leqq$. We put $u \leqq v$ whenever one of the following three conditions is satisfied

(i) $u=v$,

(ii) $u, v \in A^{*} \cup A^{\omega}$ and $\exists w[u \cdot w=v]$,

(iii) $v \in A^{*} \cdot\{\perp\}$ and $u \leqq \operatorname{strip}(v)$.

c) The function length: $A^{\text {st }} \rightarrow \mathbb{N} \cup\{\infty\}$. We put length $(u)$ as usual for $u \in A^{*}$, length $(u)=\infty$ for $u \in A^{\omega}$, and length $(u)=$ length $\left(u^{\prime}\right)+1$ for $u=u^{\prime} \perp, u^{\prime} \in A^{*}$.

d) $\mathrm{A} \leqq$-chain $\left(u_{i}\right)_{i}$ is a sequence $u_{0}, u_{1}, \ldots$, such that $u_{i} \leqq u_{i+1}, i=0,1, \ldots$. The least upper bound of the $\leqq$-chain $\left(u_{i}\right)_{i}$ is denoted by $\sup u_{i}$.

e) The $\leqq$-truncation $u(n)$ : if length $(u) \geqq n, u(n)$ denotes the prefix of $u$ of length $n$. If length $(u)<n, u(n)=u$.

f) The stream order $\sqsubseteq$ : We put $u \sqsubseteq v$ whenever one of the following two conditions is satisfied:

(i) $u=v$,

(ii) $u \in A^{*} \cdot\{\perp\}$ and $\operatorname{strip}(u) \leqq v$. 
g) A $\sqsubseteq$-chain $\left(u_{i}\right)_{i}$ is a sequence $u_{0}, u_{1}, \ldots$, such that $u_{i} \sqsubseteq u_{i+1}, i=0,1, \ldots$. The least upper bound of the $\subseteq$-chain $\left(u_{i}\right)_{i}$ is denoted by $\lim u_{i}$.

h) The $\sqsubseteq$-truncation $u[n]$. If length $(u) \geqq n$, we put $u[n]=u(n)$, if $u(n) \in$ $A^{*} \cdot\{\perp\}$, and $u[n]=u(n) \cdot \perp$, otherwise. If length $(u)<n$, we put $u[n]=u$.

Remarks. 1) Properly speaking, the concatenation of two streams as used in $\mathrm{b}$ (ii) has not yet been defined. It is in fact implicit in Definition 3.10 below.

2) A chain $\left(u_{i}\right)_{i}$ (either $\leqq-$ or $5-$-) such that $u_{i} \neq u_{i+1}$, for infinitely many $i$, is called infinitely often increasing (i.o.i.). A chain which is not i.o.i. is called stabilizing. In that case, there is an index $i_{0}$ such that $u_{i}=u_{i_{0}}$, all $i \geqq i_{0}$, and we say that $\left(u_{i}\right)_{i}$ stabilizes in $u_{i_{0}}$.

3) Do not confuse $\sup _{i} u_{i}, \operatorname{lub}_{i} u_{i}, \lim _{i} u_{i}$.

The following first results are easily shown:

Lemma 3.4. a) $\left(A^{\mathrm{st}}, \leqq, \varepsilon\right)$ is a cpo. For $a \leqq$-chain $\left(u_{i}\right)_{i}$, we have $u=\sup u_{i}$ iff

- either $\left(u_{i}\right)_{i}$ is i.o.i. and $u \in A^{\omega}$ is such that $u_{i} \leqq u$, for all $i \geqq 0$,

- or $\left(u_{i}\right)_{i}$ stabilizes in $u$.

b) $\forall u, v, w[((u \leqq w) \wedge(v \leqq w)) \Rightarrow((u \leqq v) \vee(v \leqq u))]$.

c) $\left(A^{\text {st }} \sqsubseteq, \perp\right)$ is a cpo. For $a \sqsubseteq$-chain $\left(u_{i}\right)_{i}$, we have $u=\operatorname{lub}_{i} u_{i}$ iff

- either $\left(u_{i}\right)_{i}$ is i.o.i., (hence) $u_{i}=u_{i}^{\prime} \cdot \perp$ for all $i,\left(u_{i}^{\prime}\right)_{i}$ is $a \leqq$-chain and $u=\sup u_{i}^{\prime}$,

- or $\left(u_{i}\right)_{i}$ stabilizes in $u$.

d) $u=\sup u(n)=\operatorname{lub} u[n]$

We proceed with the definition of the distance $d$ between streams:

Definition 3.5. The mapping $d: A^{\text {st }} \times A^{\text {st }} \rightarrow[0,1]$ is defined by

$$
d(u, v)=2^{-\sup \{n: u(n)=v(n)\}}
$$

with the convention that $2^{-\infty}=0$.

The following theorem is fundamental for the metric framework:

Theorem $3.6[21] .\left(A^{\mathrm{st}}, d\right)$ is a complete and compact metric space.

We next turn to the development of an order-theoretic and metric structure for sets of streams

Definition 3.7. Let $X, Y \in \mathbf{S}$.

a) $X(n)=\{u(n): u \in X\}, X[n]=\{u[n]: u \in X\}$.

b) $X \subseteq_{s} Y$ is the Smyth preorder (Definition 2.15) induced by the stream order $\subseteq$ on $A^{\text {st }}$.

c) $\min (X)=\{u: u \in X$ and for all $v \in X[v \sqsubseteq u \Rightarrow v=u]\}$.

d) Let $\mathbf{S}_{n c}$ denote the collection of all nonempty closed sets of streams. $\hat{d}(X, Y)$ denotes the Hausdorff distance (Definition 2.5) on $\mathbf{S}_{n c}$. 
e) We use $\mathbf{S}_{f}$ and $\mathbf{S}_{n c f}$ to denote the collection of all flat (Definition 2.14a) and of nonempty closed (Definition 2.14b) and flat sets of streams, respectively.

f) For a $\subseteq$-chain $\left(X_{i}\right)_{i}$ we denote its least upper bounded by $\sqcup X_{i}$.

The following theorem states, essentially, that $\mathbf{S}_{f}$ and $\mathbf{S}_{n c}$ are the structures we want. (Note, however, that we shall later specialize $\mathbf{S}_{f}$ to $\mathbf{S}_{n c f}$ to ensure continuity of the semantic operators.)

Theorem 3.8. a) $X$ is $\sqsubseteq$-closed in $\left(A^{\text {st }}, \subseteq, \perp\right)$ iff $X$ is $d$-closed in $\left(A^{\text {st }}, d\right)$.

b) For any $X, X_{1}, X_{2}$ in $\mathbf{S}$ we have

(i) $X \sqsubseteq_{s} \min (X)$ and $\min (X) \sqsubseteq_{s} X$

(ii) $X_{1} \sqsubseteq_{s} X_{2} \Leftrightarrow \min \left(X_{1}\right) \sqsubseteq_{s} \min \left(X_{2}\right)$

(iii) $(\min (X))[n]=\min (X[n])$.

c) $\left(\mathbf{S}_{f}, \subseteq_{s},\{\perp\}\right)$ is a cpo. For $\left(X_{n}\right)_{n} a \subseteq_{S^{-}}$chain we have

$$
\bigcup_{n} X_{n}=\left\{u: \underset{n}{u=\operatorname{lub}_{n}} u_{n},\left(u_{n}\right)_{n} a \sqsubseteq \text {-chain with } u_{n} \in X_{n}\right\} .
$$

d) $\left(\mathbf{S}_{n c}, \hat{d}\right)$ is a complete (and compact) metric space.

e) For $X, Y \in \mathrm{S}_{n c}, \hat{d}(X, Y)=2^{-\sup \{n: X(n)=Y(n)\}}$ with the convention that $2^{-\infty}=0$.

f) For $X \in \mathbf{S}_{n c},(X(n))_{n}$ is a Cauchy sequence in $\left(\mathbf{S}_{n c}, \hat{d}\right)$, and $X=\lim X(n)$.

g) For $X \in \mathbf{S}_{f},(X[n])_{n}$ is a $\sqsubseteq_{s^{-c h a i n}}$ in $\left(\mathbf{S}_{f}, \sqsubseteq_{s},\{\perp\}\right)$.

Proof. These result are, essentially, from [18, 19] and [2]; see also [3], [20] for related references and results.

Having defined our fundamental structures, we next arrive at the definition of the various semantic operators which we will have as counterparts of the syntactic operators: , $\cup, \|$. Once these have been defined satisfactorily, we shall have completed the preparations for the semantic definitions. Recursion will be dealt with by the familiar (least) fixed point technique, for which the relevant apparatus will then be available.

We define the semantic operators directly for $X, Y \in \mathbf{S}$, rather than going through a two stage process in which the operators are first defined on $A^{\text {st }}$. This is for convenience rather than out of necessity.

We first deal with the case that $X, Y$ consist of finite words only. Let $\mathbf{S}_{\text {fin }}$ be short for $\mathfrak{P}\left(A^{*} \cup A^{*} \cdot\{\perp\}\right)$.

Definition 3.9. We define $o \underline{p}^{\mathrm{fin}}: \mathbf{S}_{\mathrm{fin}} \times \mathbf{S}_{\mathrm{fin}} \rightarrow \mathbf{S}_{\mathrm{fin}}$, where $o \underline{p}^{\mathrm{fin}} \in\{\cdot, \cup, \|\}$. We let $X, Y$ range over $\mathbf{S}_{\text {fin }}$.

a) We assume as known the operator of prefixing which for $a \in A, u \in A^{*} \cup$ $A^{*} \cdot\{\perp\}$, delivers $a \cdot u$.

b) $a \cdot X=\{a \cdot u: u \in X\}$.

c) $X \cdot Y=\bigcup\{u \cdot Y: u \in X\}$, where $u \cdot Y$ is defined (inductively) by

$$
\varepsilon \cdot Y=Y, \perp \cdot Y=\{\perp\},(a u) \cdot Y=a \cdot(u \cdot Y) .
$$

d) $X \cup Y$ is the set-theoretic union of $X$ and $Y$. 
e) $X \| Y=(X \Perp Y) \cup(Y \Perp X)$; moreover, $X \Perp Y=\bigcup\{u \llbracket Y: u \in X\}$, where $u \Perp Y$ is defined (inductively) by $\varepsilon \llbracket Y=Y, \perp \Perp Y=\{\perp\},(a u) \llbracket Y=a \cdot(\{u\} \| Y)$.

Remark. $\Perp$ or 'left merge' stems from ACP, cf. [9]. $X \Perp Y$ denotes the interleaved execution of $X$ and $Y$ where the first step is taken from $X$.

Next, we define the metric and (Smyth-) order-theoretic operators $o p^{\mathscr{M}}$ and $o \underline{p}^{\mathscr{S}}$, where $o \underline{p}^{\mathscr{M}}, o \underline{p}^{\mathscr{S}} \in\{\cdot, \cup, \|\}$, for the general case, i.e., for $X, Y$ which do not necessarily consist of finite words only. Note that $o p^{\mathscr{S}}$ is defined on $\mathbf{S}_{n c f}$ rather than on all of $\mathbf{S}_{f}$. This is necessary to ensure continuity of $\underline{o p} \underline{\mathcal{S}}^{\mathscr{S}} \in\{\cdot, \|\}$ (see below).

Definition 3.10. a) op ${ }^{\mathscr{M}}: \mathbf{S}_{n c} \times \mathbf{S}_{n c} \rightarrow \mathbf{S}_{n c}$ is defined by

$$
X \underline{o p}^{\wedge} Y=\lim _{n}\left(X(n) \underline{o p^{f i n}} Y(n)\right) .
$$

b) $\underline{o p^{\mathscr{S}}}: \mathbf{S}_{n c f} \times \mathbf{S}_{n c f} \rightarrow \mathbf{S}_{n c f}$ is defined by

$$
\begin{array}{ll}
X \underline{o p}^{\mathscr{S}} Y=\min \left(X \underline{o p}^{\mathrm{fin}} Y\right), & \text { for } X, Y \in \mathbf{S}_{\mathrm{fin}} \cap \mathbf{S}_{n c f}, \\
X \underline{\underline{p}}^{\mathscr{S}} Y=\sqcup\left(X[n] \underline{p}^{\mathscr{S}} Y[n]\right), & \text { for } X, Y \in \mathbf{S}_{n c f} .
\end{array}
$$

The following theorem expresses well-definedness, (monotonicity and ) $\sqsubseteq s^{-}$ and $d$-continuity of the respective operators.

Theorem 3.11. a) The operators op ${ }^{\prime \prime}$ and $o p^{\mathscr{S}}$ are well-defined. In particular, they take (pairs of) nonempty closed (and flat) sets to nonempty closed (and flat) sets.

b) The operators $o^{\mathscr{S}}$ are $\sqsubseteq \mathrm{s}^{\text {-monotonic. }}$

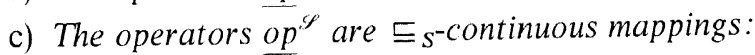

$$
\mathbf{S}_{n c f} \times \mathbf{S}_{n c f} \rightarrow \mathbf{S}_{n c f} .
$$

d) The operators $\underline{\text { op }}^{\mathscr{M}}$ are d-continuous mappings

$$
\mathrm{S}_{n c} \times \mathrm{S}_{n c} \rightarrow \mathrm{S}_{n c} \text {. }
$$

Proof. The results for $o p^{\mathscr{S}}$ are from $[18,19]$. For $o \underline{p}^{\mathscr{M}}$ the result follows from [2] and Proposition 2.9 (equivalence of $b$ ) and c)).

Remark. The sets $\left(X_{n}\right)_{n},\left(Y_{n}\right)_{n}$ defined by $X_{n}=\left\{u \in a^{*}:\right.$ length $\left.(u) \geqq n\right\}, n=0,1, \ldots$, and $Y_{n}=\left\{a^{\omega}\right\}, n=0,1, \ldots$, show that the operators $o p^{\mathscr{S}} \in\{\cdot, \|\}$ are, in general, discontinuous in the case that they are not restricted to $\mathbf{S}_{n c f} \times \mathbf{S}_{n c f}$.

We are almost ready to present the definitions of the semantic functions $\mathscr{S}$ and $\mathscr{M}$. As final preparation, we need one further syntactic notion, viz. that of guarded statements. The reason for this is that the semantics based on the metric approach is valid only for statements satisfying the guardedness requirement. (Specifically, the metric treatment of the recursive construct requires this condition to be satisfied.) Intuitively, a statement $s$ is guarded when all its recursive substatements $\mu x[t]$ satisfy the condition that (recursive) occurrences of $x$ in $t$ are 'semantically preceded' by some statement. More precisely, we have 
Definition 3.12 (guarded statements). a) We first define the notion of an occurrence of a variable being exposed in $s$. The definition is by structural induction on $s$

1. $x$ is exposed in $x$.

2. If an occurrence of $x$ is exposed in $s_{1}$, then it is exposed in $s_{1} ; s_{2}, s_{1} \| s_{2}$, $s_{2} \| s_{1}, s_{1} \cup s_{2}, s_{2} \cup s_{1}$ and $\mu y\left[s_{1}\right]$ for $y \neq x$.

b) A statement $s$ is defined to be guarded if for all its recursive substatements $\mu x[t], t$ contains no exposed occurrences of $x$.

Examples. 1. In the statement $x ; a \cup b ; x$ the first occurrence of $x$ is exposed and the second is not.

2. $\mu x[a ;(x \| b)]$ is guarded, but $\mu x[x], \mu y[y \| b]$ and $\mu y[\mu x[y]]$, as well as any statement containing these, are not.

We have now arrived at the definition of the two semantics for $\mathscr{L}$. Let $\Gamma_{\ldots}=\mathfrak{P}_{\mathrm{var}} \rightarrow \mathrm{S}_{\ldots}$, and let $\gamma \in \Gamma_{\ldots}$. (Here ... ranges over $\{n c, n c f\}$.) We use the notation $\left(\gamma^{\prime}=\right) \gamma\langle X \mid x\rangle$ for a variant of $\gamma$, which is like $\gamma$ but for its value in $x$ which equals $X$ (i.e., $\gamma^{\prime}(y)=\gamma(y)$ for $y$ F $x$ and $\gamma^{\prime}(x)=X$ ). We use $o p$ without superscript to range over the syntactic operators $\{;, \cup, \|\}$ and $o p^{\cdots}$ with superscript ... to range over the corresponding semantic operators.

Definition 3.13 (two denotational semantics). a) The mapping $\mathscr{S}: \mathscr{L} \rightarrow\left(\Gamma_{n c f} \rightarrow \mathbf{S}_{n c f}\right)$ is defined by

(i) $\mathscr{S} \llbracket a \rrbracket(\gamma)=\{a\}$,

(ii) $\mathscr{S} \llbracket s_{1} \underline{o p} s_{2} \rrbracket(\gamma)=\mathscr{S} \llbracket s_{1} \rrbracket(\gamma) \underline{o p} \mathscr{\mathscr { S }} \mathscr{S} \llbracket s_{2} \rrbracket(\gamma)$,

(iii) $\mathscr{S} \llbracket x \rrbracket(\gamma)=\gamma(x)$,

(iv) $\mathscr{S} \llbracket \mu x[s] \rrbracket(\gamma)=\underset{n}{\sqcup} X_{n}$, where $X_{0}=\{\perp\}$ and $X_{n+1}=\mathscr{S} \llbracket s \rrbracket\left(\gamma\left\langle X_{n} / x\right\rangle\right)$.

b) The mapping $\mathscr{M}: \mathscr{L} \rightarrow\left(\Gamma_{n c} \rightarrow \mathbf{S}_{n c}\right)$ is defined by

(i) $\mathscr{M} \llbracket a \rrbracket(\gamma)=\{a\}$,

(ii) $\mathscr{M} \llbracket s_{1} \underline{o p} s_{2} \rrbracket(\gamma)=\mathscr{M} \llbracket s_{1} \rrbracket(\gamma) \underline{p}^{\mathscr{M}} \mathscr{M} \llbracket s_{2} \rrbracket(\gamma)$,

(iii) $\mathscr{M} \llbracket x \rrbracket(\gamma)=\gamma(x)$,

(iv) $\mathscr{M} \llbracket \mu x[s] \rrbracket(\gamma)=\lim X_{n}$, where $X_{0}=\{\perp\}$ and $X_{n+1}=\mathscr{M} \llbracket s \rrbracket\left(\gamma\left\langle X_{n} / x\right\rangle\right)$.

The following facts support this definition

Theorem 3.14. a) The function $\Phi=\lambda X \cdot \mathscr{S} \llbracket s \rrbracket(\gamma\langle X / x\rangle)$ is a $\varsigma_{s}$-continuous mapping: $\mathbf{S}_{n c f} \rightarrow \mathbf{S}_{n c j}$ and, for $\left(X_{n}\right)_{n}$ as in clause a (iv), $\sqcup X_{n}=\mu \Phi$.

b) Assume $s$ guarded. The function $\Psi=\lambda X \cdot \mathscr{M} \llbracket s \rrbracket(\gamma\langle X / x\rangle)$ is a contracting mapping: $\mathbf{S}_{n c} \rightarrow \mathbf{S}_{n c}$, and, for $\left(X_{n}\right)_{n}$ as in clause b (iv), $\lim X_{n}$ yields the unique fixed point of $\Psi$.

Remark. For the contractivity property in part $\mathrm{b}$ of this theorem, the guardedness of $s$ is necessary. For the semantic function $\mathscr{S}$, the situation is the following:

(i) For (closed and) guarded $s$ we have that $\mathscr{S} \llbracket s \rrbracket(\gamma) \subseteq A^{*} \cup A^{\omega}$. This is a consequence of an analogous fact for $\mathscr{M}$ (see end of Sect. 5) and the equality $\mathscr{S}=\mathscr{M}$ (Theorem 3.15 below). 
(ii) For unguarded $s, \mathscr{S} \llbracket s \rrbracket(\gamma)$ will involve streams ending in $\perp$. For example $\mathscr{S} \llbracket(a ; \mu x[b \| x]) \cup c \rrbracket(\gamma)=\{a \perp, c\}$ and $\mathscr{S} \llbracket(a ; \mu x[b \| x]) \cup(a ; c) \rrbracket(\gamma)=\{a \perp\}$. This follows from (the treatment of recursion and) the flattening operator min in the definition of $\underline{o p}$ (in the clause $X \underline{p}^{\mathscr{S}} Y=\min \left(X \underline{o p^{\mathrm{fin}}} Y\right), X, Y$ with finite words only).

Our aim in the next section will be to prove the

Theorem 3.15. For each closed and guarded $s \in \mathscr{L}$

$$
\mathscr{S} \llbracket s \rrbracket=\mathscr{M} \llbracket s \rrbracket .
$$

In order to establish this result, we have to study the relationship between the two structures $\mathbf{S}_{n c f}$ as a cpo are $\mathbf{S}_{n c}$ as a metric space in more detail, as we shall do in Sect. 4.

\section{Relating the Semantic Domains}

The first main result of this section states that, for $\left(X_{i}\right)_{i}$ a $\subseteq_{s}$-chain in $\mathbf{S}_{n c f}$, $\left(X_{i}\right)_{i}$ is also a Cauchy sequence (in $\mathbf{S}_{n c}$ ), and $\lim X_{i}=\sqcup X_{i}$. This result is, clearly, fundamental for the proof of

$$
\mathscr{U} \llbracket s \rrbracket=\mathscr{S} \llbracket s \rrbracket,
$$

for $s$ a recursive construct. The second part of the section is devoted to a number of properties of the min-operator. We first prove that $\min$ is $d$-continuous. Next, we use this - and various other properties of $\min$ - to prove that, if $\min \left(X_{i}\right)=Y_{i}$, $X_{i} \in \mathbf{S}_{n c}, Y_{i} \in \mathbf{S}_{n c f}, i=1,2$, then $\min \left(X_{1} o \underline{p}^{\mathscr{M}} X_{2}\right)=Y_{1} \underline{o p^{\mathscr{S}}} Y_{2}$. The latter result is crucial for the derivation of $(*)$ for $s$ of the form $s_{1}$ op $s_{2}$.

We begin with an auxiliary lemma.

Lemma 4.1 (interpolation). a) Let $\left(X_{i}\right)_{i}$ be $a \sqsubseteq s^{\text {-chain in }} \mathbf{S}_{n c j}$. For each $\sqsubseteq$-chain $\left(u_{i_{j}}\right)_{j}$, with $u_{i_{j}} \in X_{i_{j}}, j=0,1, \ldots$, there exists $a \sqsubseteq$-chain $\left(u_{i}\right)_{i}$, with $u_{i} \in X_{i}, i=0,1, \ldots$, which has $\left(u_{i_{j}}\right)_{j}$ as a subsequence.

b) Let $\left(X_{i}\right)_{i}$ be $a \sqsubseteq{ }_{s}$-chain in $\mathbf{S}_{n c s}$. For each convergent sequence $\left(u_{i j}\right)_{j}$, with $u_{i_{j}} \in X_{i_{j}}, j=0,1, \ldots$, there exists a convergent sequence $\left(u_{i}\right)_{i}, u_{i} \in X_{i}$, containing $\left(u_{i_{j}}\right)_{j}$ as a subsequence (and, consequently, $\lim _{j} u_{i_{j}}=\lim _{i} u_{i}$ ).

Proof. a) It is, clearly, sufficient to prove that, if $X \sqsubseteq_{S} Y \sqsubseteq_{S} Z, X, Y, Z \in \mathrm{S}_{n c f}$, and $u \in X, w \in Z$ with $u \subseteq w$, then there exists $v \in Y$ with $u \subseteq v \sqsubseteq w$. By the definition of $\subseteq_{S}$ we find $v_{1} \in Y$ such that $v_{1} \sqsubseteq w$ and $u_{1} \in X$ such that $u_{1} \sqsubseteq v_{1} \sqsubseteq w$. Since both $u \sqsubseteq w$ and $u_{1} \sqsubseteq w$ we have $u_{1} \sqsubseteq u$ or $u \sqsubseteq u_{1}$. Since $X$ is flat we have $u_{1}=u$, and we see that $v_{1}$ is the desired element in $Y$.

b) Let $u_{i_{j}}=v_{j} w_{j}$, where $\left(v_{j}\right)$ is a $\leqq$-chain and $\sup _{j} v_{j}=\lim _{j} u_{i_{j}}$. Consider, for some fixed $j, u_{i_{j}}$ and $u_{i_{j+1}}$, and suppose $i_{j+1}-i_{j}>1$. So, for some $i, i_{j}<i<i_{j+1}$. We can find an element $u_{i}$ such that $u_{i}=v_{j} w_{j}^{\prime}$ for some $w_{j}^{\prime}$. This can be seen as follows: Since $X_{i} \subseteq_{S} X_{i_{j+1}}$ there must be an element $u_{i}$ such that $u_{i} \subseteq u_{i_{j+1}}$ $=v_{j+1} w_{j+1}=v_{j} w_{j+1}^{\prime}$, for some $w_{j+1}^{\prime}$. If $u_{i} \in A^{*} \cup A^{\omega}$, the result is immediate. 
Now let $u_{i}=u \perp$. If $u$ is such that $v_{j} \leqq u$, we have finished. If $u<v_{j}$ we argue as follows: Since $X_{i_{j}} \subseteq_{s} X_{i}$, there must be some $u_{i_{j}}^{\prime}$ such that $u_{i_{j}}^{\prime} \sqsubseteq u \perp \sqsubseteq v_{j} w_{j}$. By flatness of $X_{i_{j}}, u_{i_{j}}^{\prime}=v_{j} w_{j}$. So $u_{i}=u \perp=u_{i_{j}}^{\prime}=v_{j} w_{j}$ as well. Hence in this case we have also found an element $u_{i}$ as desired. Consequently, we are always able to interpolate the converging sequence $\left(u_{i_{j}}\right)_{j}$ to one of the form $\left(u_{i}\right)_{i}$, where $u_{i} \in X_{i}, i=0,1, \ldots$.

The next lemma is also auxiliary, and relies essentially on the compactness of $\left(A^{\mathrm{st}}, d\right)$.

Lemma 4.2. Let $X_{1}, X_{2} \in \mathrm{S}_{n c}$. (At least) one of the following two conditions holds:

1. There exists $\bar{u}_{1} \in X_{1}$ such that

$$
\left.\hat{d}\left(X_{1}, X_{2}\right)=\sup _{u_{1} \in X_{1}} d^{\prime}\left(u_{1}, X_{2}\right)=d^{\prime}\left(\bar{u}_{1}, X_{2}\right) \quad \text { (see Definition } 2.5 \text { for } d^{\prime}\right)
$$

2. There exists $\bar{u}_{2} \in X_{2}$ such that

$$
\hat{d}\left(X_{1}, X_{2}\right)=\sup _{u_{2} \in X_{2}} d^{\prime}\left(u_{2}, X_{1}\right)=d^{\prime}\left(\bar{u}_{2}, X_{1}\right) .
$$

Proof. Direct from the fact that a (real-valued) continuous function on a compact set attains its maximum.

We next state two important properties which relate $\sqcup X_{i}$ and $\lim X_{i}$.

Lemma 4.3. Each $\sqsubseteq s^{-c h a i n}\left(X_{i}\right)_{i}$, with $X_{i}$ in $\mathbf{S}_{n c f}$, is a Cauchy sequence (in $\left.\mathbf{S}_{n c}\right)$.

Proof (cf. [24]). Let $\left(X_{j}\right)_{j}$ be a $\sqsubseteq_{s}$-chain in $S_{n c f}$. We define the set $\lim X_{j}$ by

$$
\lim _{j} X_{j}=\left\{u \mid u=\lim _{j} u_{j}, u_{j} \in X_{j} \text { and }\left(u_{j}\right)_{j} \text { a Cauchy sequence }\right\} .
$$

(Note that this definition does not require that $\left(X_{j}\right)_{j}$ is a Cauchy sequence.) We first prove that the set $\lim _{j} X_{j}$ is nonempty and closed. By ([18], p. 91-93),
the set

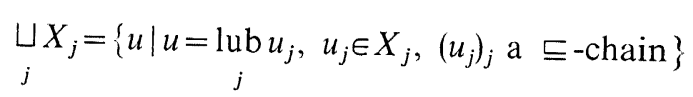

is nonempty if all $X_{j}$ are nonempty. Since every $\sqsubseteq$-chain $\left(u_{j}\right)_{j}$ in $A^{\text {st }}$ is also a Cauchy sequence such that $\lim u_{j}=\operatorname{lub} u_{j}$, we clearly have that $\sqcup X_{j} \subseteq \lim X_{j}$; hence, $\lim X_{j}$ is nonempty. In order to prove that ${ }^{j} \lim X_{j}$ is closed. Assume that $\left(u_{i}\right)_{i}$ is a Cauchy sequence in $\lim _{j} X_{j}$. Then, for each $i, u_{i}=\underset{j}{\lim } u_{i, j}$ for $\left(u_{i, j}\right)_{j}$ a Cauchy sequence with $u_{i, j} \in X_{j}, j=0,1, \ldots$ Following an argument as in $([16]$, Proposition 4.3 , p. 303) we can find a sequence $\left(u_{j}\right)_{j}$ of indices such that $\left(u_{j, n_{j}}\right)_{j}$ is also a Cauchy sequence, and $\lim u_{i}=\lim _{j} u_{j, n_{j}} \in \lim _{j} X_{n_{j}} \subseteq \lim _{j} X_{j}$ (the inclusion 
holds by interpolation). We shall now show that

$$
\hat{d}\left(X_{i}, \lim _{j} X_{j}\right) \rightarrow 0 \quad \text { as } i \rightarrow \infty,
$$

thus proving that $\left(X_{i}\right)_{i}$ is a Cauchy sequence. We shall only exhibit the proof that

$$
\sup _{u \in X_{i}} d^{\prime}\left(u, \lim _{j} X_{j}\right) \rightarrow 0 \quad \text { as } i \rightarrow \infty \text {. }
$$

By Lemma 4.2 there exist $u_{i}$ such that $\sup d^{\prime}\left(u, \lim X_{j}\right)=d^{\prime}\left(u_{i}, \lim X_{j}\right)$. By com-

$$
u \in X_{i} \quad j \quad j
$$

pactness, $\left(u_{i}\right)_{i}$ has a converging subsequence $\left(u_{i_{j}}\right)_{j}$. Suppose $\lim u_{i_{j}}=\bar{u}$. By Lemma

$4.1 \mathrm{~b}$ there are interpolating $u_{i}^{\prime} \in X_{i}$ such that $\left(u_{i}^{\prime}\right)_{i}$ contains $\left(u_{i_{j}}\right)_{j}$ as a subsequence. So $\bar{u} \in \lim X_{j}$. Now let $\varepsilon>0$ and choose $i_{k}$ such that $d\left(u_{i_{k}}, \bar{u}\right)<\varepsilon$. Then, for each $j \geqq i_{k}$,

$$
\begin{aligned}
& \sup _{u \in X_{j}} d^{\prime}\left(u, \lim _{j} X_{j}\right) \leqq\left(\text { since } X_{i_{k}} \sqsubseteq_{S} X_{j}\right) \\
& \sup _{u \in X i_{k}} d^{\prime}\left(u, \lim _{j} X_{j}\right)= \\
& d^{\prime}\left(u_{i_{k}}, \lim _{j} X_{j}\right) \leqq\left(\text { since } \bar{u} \in \lim _{j} X_{j}\right) \\
& d\left(u_{i_{k}}, \bar{u}\right)<\varepsilon .
\end{aligned}
$$

For $\left(X_{j}\right)_{j}$ a $\subseteq_{s}$-chain in $S_{n c f}$, we now know that $\left(X_{j}\right)_{j}$ is also a Cauchy sequence. The next theorem answers the natural question 'is it the case that $\sqcup X_{j}=\lim X_{j}$ ?' affirmatively.

Theorem 4.4. Let $\left(X_{j}\right)_{j}$ be $a \sqsubseteq s^{-c h a i n}$ in $\mathbf{S}_{n c f}$. Then

$$
\sqcup_{j} X_{j}=\lim _{j} X_{j}
$$

Proof. Recall that

$$
\begin{gathered}
\lim _{j} X_{j}=\left\{u \mid u=\lim _{j} u_{j}, u_{j} \in X_{j},\left(u_{j}\right)_{j} \text { a Cauchy sequence }\right\} \\
\underset{j}{\sqcup X_{j}}=\left\{u \mid u=\operatorname{lub}_{j} u_{j}, u_{j} \in X_{j},\left(u_{j}\right)_{j} \text { a } \sqsubseteq \text {-chain }\right\} .
\end{gathered}
$$

As before, $\sqcup X_{j} \subseteq \lim _{j} X_{j}$. There remains the proof that $\lim _{j} X_{j} \subseteq \sqcup X_{j}$. Take some $u=\lim u_{j} \in \lim X_{j}$. First we assume that the sequence $\left(u_{j}\right)_{j}$ stabilizes at some $u_{i_{0}}$. By the definition of $\subseteq_{s}$, there is a $\sqsubseteq$-chain $u_{0}^{j} \sqsubseteq u_{1}^{\prime} \sqsubseteq \ldots \sqsubseteq u_{i}^{\prime}=u_{i+1}^{\prime}=\ldots$ with $u_{i}^{\prime}=u_{i_{0}}, i \geqq i_{0}$. thus, $u=u_{i_{0}}^{\prime}=$ lub $u_{i}^{\prime}$. Now take the case that $\left(u_{j}\right)_{j}$ does not stabilize. Thus, $u \in A^{\omega}$. We consider, for some fixed $j$, the set $X_{j}$. Since $X_{j} \sqsubseteq{ }_{s} X_{j+i}, i \geqq 0$, there must be elements $u_{j}^{(i)} \in X_{j}$ such that $u_{j}^{(i)} \sqsubseteq u_{j+i}$. Let $V_{j}={ }^{\text {df }}\left\{u_{j}^{(i)} \mid i \geqq 0\right\}$. If $V_{j}$ 
is infinite, it must contain an infinite (i.o.i.) convergent subsequence $\left(u_{j}^{\left(i_{k}\right)}\right)_{k}$. Since $V_{j} \subseteq X_{j}$ and $X_{j}$ is closed, $X_{j}$ must contain $\lim u_{j}^{\left(i_{k}\right)}$. Since, for each $k, u_{j}^{\left(i_{k}\right)} \sqsubseteq u_{j+i_{k}}$, and since the sequence $\left(u_{j}^{\left(i_{k}\right)}\right)_{k}$ is i.o.i., we have that $\lim u_{j}^{\left(i_{k}\right)}=u$. Thus, for $V_{j}$ infinite we infer that $u \in X_{j}$. We now distinguish two cases:

Case $1 . V_{j}$ is infinite for almost all $j$, say for all $j \geqq j_{0}$. We can then construct the chain

$$
u_{0}^{\prime} \sqsubseteq u_{1}^{\prime} \sqsubseteq \ldots \sqsubseteq u_{j_{0}}^{\prime} \sqsubseteq u_{j_{0}+1}^{\prime} \sqsubseteq \ldots
$$

with $u_{j_{0}+l}^{\prime}=u, l \geqq 0$. Thus, $u=\operatorname{lub} u_{n}^{\prime}$, and we are done.

Case 2. There are infinitely many finite $V_{j}$, say $V_{j}$ is finite for all $j$ in the index set $J$. Consider such a finite $V_{j}$. Since $V_{j}$ contains a finite number of elements approximating an infinite number of streams $\left(u_{j+i}\right.$, all $\left.i \geqq 0\right), V_{j}$ must contain a stream of the form $u_{j} \perp$ which approximates an infinite number of the $u_{j+i}$ $(i \geqq 0)$. This must be the case for all $j \in J$. Clearly, $u_{j} \perp \subseteq u$ for all $j \in J$. Thus, for $j<j^{\prime}$, either $u_{j} \perp \sqsubseteq u_{j^{\prime}} \perp$ or $u_{j^{\prime}} \perp \sqsubseteq u_{j} \perp$. However, since the $\left(X_{i}\right)_{i}$ form a

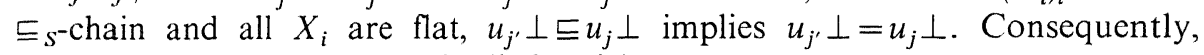
$\left(u_{j} \perp\right)_{j \in J}$ is a $\subseteq$-chain. We again distinguish two cases.

Subcase 2.1. The chain $\left(u_{j} \perp\right)_{j \in J}$ is i.o.i. Then, after applying the interpolation lemma, we obtain the chain

$$
u_{0}^{\prime} \sqsubseteq u_{1}^{\prime} \sqsubseteq \ldots \sqsubseteq u_{j_{1}} \perp=u_{j_{1}}^{\prime} \sqsubseteq \ldots \sqsubseteq u_{j_{2}} \perp=u_{j_{2}}^{\prime} \sqsubseteq \ldots
$$

with $u_{n}^{\prime} \in X_{n}$ and $u=\operatorname{lub} u_{n}^{\prime}$.

Subcase 2.2. The chain $\left(u_{j} \perp\right)_{j \in J}$ stabilizes at some $\bar{j}$ :

$$
u_{j_{1}} \perp \sqsubseteq u_{j_{2}} \perp \sqsubseteq \ldots \sqsubseteq u_{j} \perp=\ldots .
$$

This implies that there must be some $k \geqq \bar{j}$, where $X_{k}$ contains both $u_{\bar{j}} \perp$ and $u_{k}$, and $u_{j} \perp \sqsubseteq u_{k}$, contradicting the flatness of $X_{k}$.

Altogether, if $\left(u_{j}\right)_{j}$ is i.o.i. and $u$ is infinite there must be a chain $\left(u_{j}^{\prime}\right)_{j}$ with $u_{j}^{\prime} \in X_{j}$ and $\operatorname{lub}_{j} u_{j}^{\prime}=u$, i.e., we have found $\underset{j}{\sqcup} \underset{j}{\perp} X_{j}$.

The second part of Sect. 4 is devoted to an analysis of various properties of the min-operator. We begin with an easy result:

Lemma 4.5. For $X, Y \in \mathbf{S}$ and op any $\subseteq_{s}$-monotonic operator: $\mathbf{S} \times \mathbf{S} \rightarrow \mathbf{S}$, we have

$$
\min (X \underline{o p} Y)=\min (\min (X) \underline{o p} \min (Y)) .
$$

Proof. Since $X \sqsubseteq_{S} \min (X) \subseteq_{S} X$, and similarly for $Y$, we have, by the monotonicity of $o p$, that

$$
X \underline{o p} Y \sqsubseteq_{s} \min (X) \underline{o p} \min (Y) \sqsubseteq_{S} X \underline{o p} Y .
$$

Thus, by the monotonicity of $\min , \min (X \underline{o p} Y) \subseteq{ }_{s} \min (\min (X) \underline{o p} \min (Y)) \sqsubseteq$ $\min (X$ op $Y)$. Since $\subseteq_{S}$ is an order on flat sets, we have the desired result. 
Next, we prove the $d$-continuity of min:

Theorem 4.6. Let $\left(X_{i}\right)_{i}$ be a Cauchy sequence in $\left(\mathbf{S}_{n c}, \hat{d}\right)$. Then $\min \left(\lim X_{i}\right)$ $=\lim \min \left(X_{i}\right)$.

Proof. We prove two inclusions.

Part 1. $\lim \min \left(X_{i}\right) \leqq \min \left(\lim X_{i}\right)$. Take some $u \in \lim \min \left(X_{i}\right)$, i.e., $u=\lim u_{i}$, $u_{i} \in \min \left(X_{i}\right) \leqq X_{i}$. Thus, $u \in \lim X_{i}$. We show that $u$ is a minimal element in $\lim X_{i}$.

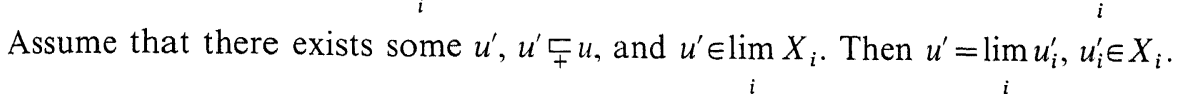
We distinguish two cases:

(i) $u^{\prime}=\lim _{i} u_{i}^{\prime}$ is infinite. This is impossible since $u_{+}^{\prime}{ }_{+} u$. ble.

(ii) $u^{\prime}=\lim _{i} u_{i}^{\prime}$ is finite. Then $u^{\prime}=u_{i_{0}}^{\prime}$ for some $u_{i_{0}}^{\prime}$. If $u^{\prime} \in A^{*}, u^{\prime} \sqsubseteq+u$ is impossi-

There remains the case that $u^{\prime}=\bar{u} \perp$ for some $\bar{u} \in A^{*}$. If $u \in A^{\omega}$ then $\exists i>i_{0}$ $\left[\left(u_{i} \in X_{i}\right) \vee\left(u_{i_{0}}^{\prime}=\right) u_{i}^{\prime} \subsetneq u_{i}\right]$. This contradicts the minimality of $u_{i}$. If $u \in A^{*} \cup$ $A^{*} \cdot\{\perp\}$, then $\lim _{i} u_{i}=u_{j_{0}}$ for some $j_{0}$. Now take $k_{0}=\max \left(i_{0}, j_{0}\right)$. Then $u_{k_{0}}^{\prime} \subsetneq u_{k_{0}}$, which again yields a contradiction.

Part 2. We prove $\min \left(\lim X_{i}\right) \cong \lim \min \left(X_{i}\right)$. Take $u \in \min \left(\lim X_{i}\right)$. Thus, $u=\lim u_{i}$, $u_{i} \in X_{i}$, amd $u$ is minimal. We now take $u_{i}^{\prime} \in \min \left(X_{i}\right)$ such that $u_{i}^{\prime} \subseteq u_{i}$, and consider $\lim u_{i}^{\prime}$.

Subcase $1 . \lim u_{i}^{\prime}$ is infinite. We can find a prefix chain $\left(v_{i}\right)_{i}$ such that $u_{i}^{\prime}=v_{i} w_{i}^{\prime}$, $u_{i}=v_{i} w_{i}$ and $w_{i}^{\prime} \subseteq w_{i}$. Moreover, $u=\lim u_{i}=\sup v_{i}=\lim u_{i}^{\prime}=u^{\prime}$. Thus, in this case $u \in \lim \min \left(X_{i}\right)$.

Subcase 2. $\lim _{i} u_{i}^{\prime}$ is finite, say $\lim _{i} u_{i}^{\prime}=u_{i_{0}}^{\prime}$. If $\exists i \forall j \geqq i\left[u_{j}=u_{j}^{\prime}\right]$ then $u_{i}=\lim _{i} u_{i}$ $=\lim u_{i}^{\prime} \in \lim \min \left(X_{i}\right)$. Otherwise, $\forall i \exists j \geqq i\left[u_{j^{\prime}}^{\prime} \subsetneq u_{j}\right]$. Since $u_{j}^{\prime}=u_{i_{0}}^{\prime}$ for $j \geqq i_{0}$, we now have that $u_{i} \neq u_{i_{0}}^{\prime}$ for infinitely many $i$, so $u=\lim u_{i} \neq u_{i_{0}}^{\prime}=\lim u_{i}^{\prime}=u^{\prime}$.

We show that this leads to a contradiction. Once more, we distinguish two subcases:

Subcase 2.1. $u=\lim u_{i}$ is finite, say $\lim u_{i}=u_{j_{0}}$. Take $k_{0}=\max \left(i_{0}, j_{0}\right)$. Then $u^{\prime}$ $=\lim u_{i}^{\prime}=u_{k_{0}}^{\prime} \sqsubseteq u_{k_{0}}=\lim u_{i}=u$. The two facts $u^{\prime} \sqsubseteq u$ and $u^{\prime} \neq u$ contradict the minimality of $u$.

Subcase 2.2. $u=\lim u_{i}$ is infinite. Then there exist $v_{i}, w_{i}$ such that $u_{i}=v_{i} w_{i},\left(v_{i}\right)_{i}$ 
is a prefix chain, and $\lim u_{i}=\sup v_{i}$. Since $\sup v_{i}$ is infinite we have $\exists j_{0} \forall j$ $\geqq j_{0}\left[u_{i_{0}}^{\prime} \subseteq v_{j}\right]$. So $u^{\prime}=\lim _{i} u_{i}^{\prime}=u_{i_{0}}^{\prime} \subseteq \sup _{i} v_{i}=u$. Again, we have $u^{\prime} \sqsubseteq u$ and $u^{\prime} \neq u$, a contradiction as in Subcase 2.1.

We are now in the position to establish the main technical result relating the operators $\underline{o p^{\mathscr{M}}}$ and $o \underline{p}^{\mathscr{S}}$.

Theorem 4.7. Let $o p^{\mathscr{M}}, \underline{o p^{\mathscr{S}}}$ be as in Definition 3.10, let $X_{1}, X_{2} \in \mathrm{S}_{n c}$ and $Y_{1}, Y_{2} \in \mathrm{S}_{n c f}$, and assume

$$
\min \left(X_{i}\right)=Y_{i}, \quad i=1,2 .
$$

Then $\min \left(X_{1} \underline{\underline{p}} \underline{\underline{M}}^{\mathscr{M}} X_{2}\right)=Y_{1} \underline{o \underline{p}^{\mathscr{P}}} Y_{2}$.

Proof. We have, successively,

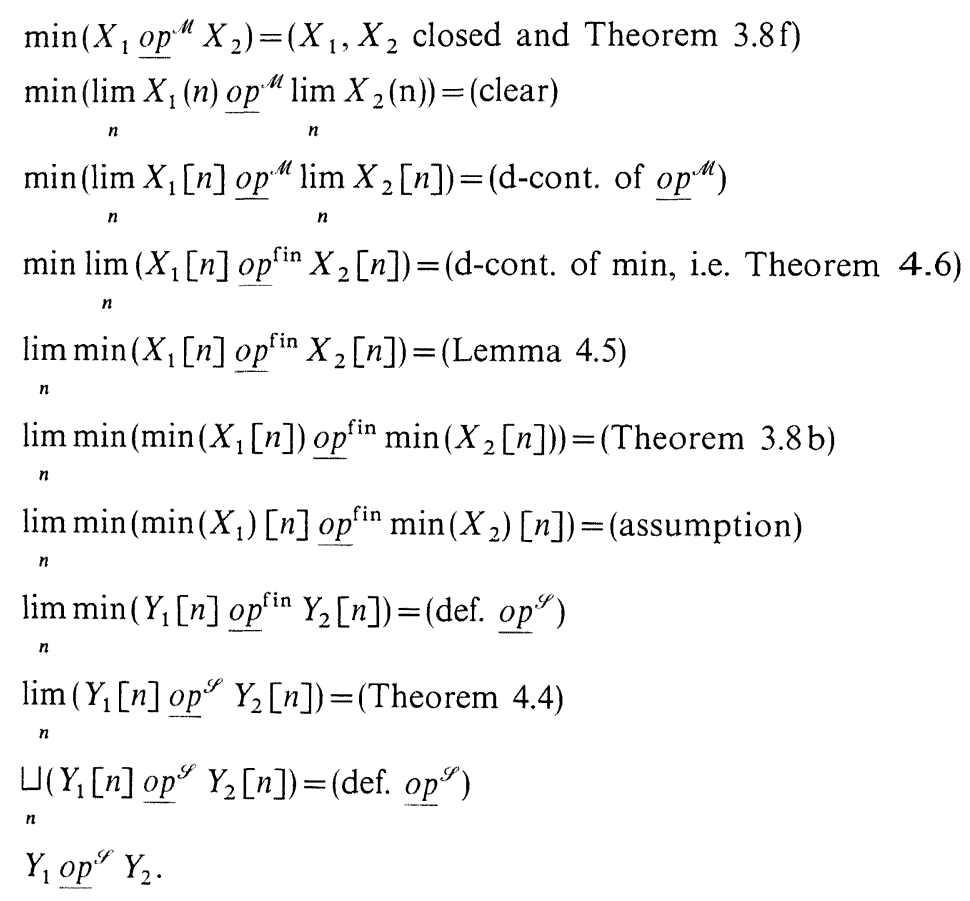

\section{Proof of the Equivalence Theorem}

In Sect. 4 we have collected all results necessary to prove the main result of this paper which we repeat here for convenience:

Theorem 3.15. For closed and guarded $s \in \mathscr{L}$

$$
\mathscr{M} \llbracket s \rrbracket=\mathscr{S} \llbracket s \rrbracket .
$$


Proof. We first prove a more general result - following a similar pattern as in [7], proof of Theorem 2.4.1 - in which $s$ is not necessarily syntactically closed (but still guarded), viz.

$$
\min \left(\mathscr{M} \llbracket s \rrbracket\left(\gamma\left\langle X_{i} / x_{i}\right\rangle_{i=1}^{n}\right)\right)=\mathscr{P} \llbracket s \rrbracket\left(\gamma\left\langle Y_{i} 0 x_{i}\right\rangle_{i=1}^{n}\right)
$$

where

(i) $\left\{x_{1}, \ldots, x_{n}\right\}$ is the set of free process variables in $s$

(ii) $\min \left(X_{i}\right)=Y_{i}, i=1,2, \ldots, n$.

We prove $(*)$ by induction on the complexity of $s$. If $s \equiv a$ the result is obvious and if $s \equiv x$ then $x \equiv x_{i}$ for some $i \in\{1, \ldots, n\}$ and the desired result follows from (ii). Next, we consider the case that $s \equiv s_{1} \underline{o p} s_{2}$, for $\underline{o p} \in\{;, \cup, \|\}$. Then

$$
\begin{aligned}
& \min \left(\mathscr{M} \llbracket s \rrbracket\left(\gamma\left\langle X_{i} / x_{i}\right\rangle_{i}\right)\right)= \\
& \min \left(\mathscr{M} \llbracket s_{1} o p s_{2} \rrbracket\left(\gamma\left\langle X_{i} / x_{i}\right\rangle_{i}\right)\right)= \\
& \min \left(\mathscr{M} \llbracket s_{1} \rrbracket\left(\gamma\left\langle X_{i} / x_{i}\right\rangle_{i}\right) \underline{p}^{\mathscr{M}} \cdot \mathscr{M} \llbracket s_{2} \rrbracket\left(\gamma\left\langle X_{i} / x_{i}\right\rangle_{i}\right)\right)=
\end{aligned}
$$

(by the induction hypothesis and Theorem 4.7)

$$
\begin{aligned}
& \mathscr{S} \llbracket s_{1} \rrbracket\left(\gamma\left\langle Y_{i} / x_{i}\right\rangle_{i}\right) \underline{o P^{\mathscr{S}}} \mathscr{S} \llbracket s_{2} \rrbracket\left(\gamma\left\langle Y_{i} / x_{i}\right\rangle_{i}\right)= \\
& \mathscr{S} \llbracket s_{1} \underline{o p} s_{2} \rrbracket\left(\gamma\left\langle Y_{i} / x_{i}\right\rangle_{i}\right)=\mathscr{S} \llbracket s \rrbracket\left(\gamma\left\langle Y_{i} / x_{i}\right\rangle_{i}\right) .
\end{aligned}
$$

Finally, consider the case that $s \equiv \mu y\left[s_{0}\right]$, for some $y$ and $s_{0}$. Without lack of generality, we assume $y \notin\left\{x_{1}, \ldots, x_{n}\right\}$. Let $Z_{0}=U_{0}=\{\perp\}$ and

$$
\begin{aligned}
& Z_{k+1}=\mathscr{M} \llbracket s_{0} \rrbracket\left(\gamma\left\langle X_{i} / x_{i}, Z_{k} / y\right\rangle_{i=1}^{n}\right), \\
& U_{k+1}=\mathscr{S} \llbracket s_{0} \rrbracket\left(\gamma\left\langle Y_{i} / x_{i}, U_{k} / y\right\rangle_{i=1}^{n}\right) .
\end{aligned}
$$

Then $\mathscr{M} \llbracket \mu y\left[s_{0}\right] \rrbracket\left(\gamma\left\langle X_{i} / x_{i}\right\rangle_{i=1}^{n}\right)=\lim Z_{k}$, and $\mathscr{S} \llbracket \mu y\left[s_{0}\right] \rrbracket\left(\gamma\left\langle Y_{i} / x_{i}\right\rangle_{i=1}^{n}\right)=\sqcup U_{k}$. We shall prove that $(* *) \min \left(\lim Z_{k}\right)=\sqcup U_{k}$. By $d$-continuity of min, the fact that $\left(U_{k}\right)_{k}$ is a Cauchy sequence and Theorem 4.4, we replace $(* *)$ by $\lim \min \left(Z_{k}\right)=\lim U_{k}$. Thus, it is sufficient to prove $(* * *) \min \left(Z_{k}\right)=U_{k}, k=0,1, \ldots$. $k$

We use induction on $k$. The case $k=0$ is clear. Next assume $(* * *)$, to prove $\min \left(Z_{k+1}\right)=U_{k+1}$, i.e.,

$$
\min \left(\mathscr{M} \llbracket s_{0} \rrbracket\left(\gamma\left\langle X_{i} / x_{i}, Z_{k} / y\right\rangle_{i=1}^{n}\right)\right)=\mathscr{S} \llbracket s_{0} \rrbracket\left(\gamma\left\langle X_{i} / x_{i}, U_{k} / y\right\rangle_{i=1}^{n}\right) .
$$

Now this follows from the main induction hypothesis (for $(*)$ ), with $s_{0}$ replacing $s$ and $n+1$ replacing $n$, and using $(* * *)$ to establish the $(n+1)$-st part of condition (ii).

We are almost finished with the proof: for closed $s$, the set of its free variables is empty, and $(*)$ specializes to

$$
\min (\mathscr{M} \llbracket s \rrbracket(\gamma))=\mathscr{S} \llbracket s \rrbracket(\gamma) .
$$


By the definition of $\mathscr{M} \llbracket s \rrbracket$ it is easily seen that, for $s$ closed and guarded, $\mathscr{M} \llbracket \llbracket \rrbracket(\gamma) \subseteq A^{*} \cup A^{\omega}$. This follows from Definition $3.14 \mathrm{~b}$, after varying its clause $3.14 \mathrm{~b}$ (iv) by taking for $X_{0}$ an arbitrary subset of $A^{*} \cup A^{\omega}$. (The choice for $X_{0}$ is immaterial anyway (see Proposition 2.4b); the choice $X_{\mathrm{O}}=\{\perp\}$ was convenient in the proof just given where we showed $Z_{0}=U_{0}$.) It is then straightforward to show that $\mathscr{M} \llbracket s \rrbracket(\gamma) \subseteq A^{*} \cup A^{\omega}$ by structural induction on $s$. Thus, $\min (\mathscr{M} \llbracket s \rrbracket(\gamma))=\mathscr{M} \llbracket s \rrbracket(\gamma)$. Altogether, we have established that, for $s$ closed and guarded, $\mathscr{M} \llbracket s \rrbracket=\mathscr{S} \llbracket s \rrbracket$, as was to be shown.

Acknowledgement. Jeff Zucker has scrutinized a first version of this paper and suggested various corrections and improvements. We are grateful to the members of the CWI concurrency project, Frank de Boer, Joost Kok, Jan Rutten, Anton Eliens, and to Erik de Vink for their comments on a first presentation of the material in this paper. We also acknowledge the meticulous reading of the paper by the referees.

\section{References}

1. Back, R.J.: A continuous semantics for unbounded nondeterminism. Theor. Comput. Sci. 23, $187-210(1983)$

2. de Bakker, J.W., Bergstra, J.A., Klop, J.W., Meyer, J.-J.Ch.: Linear time and branching time semantics for recursion with merge. Theor. Comput. Sci. 34, 135-156 (1984)

3. de Bakker, J.W., Kok, J.N., Meyer, J.-J.Ch., Olderog, E.-R., Zucker, J.I.: Contrasting themes in the semantics of imperative concurrency. In: Current Trends in Concurrency: Overviews and Tutorials (J.W. de Bakker, W.P. de Roever, G. Rozenberg, eds.), LNCS 224, pp. 51-121. BerlinHeidelberg-New York: Springer 1986

4. de Bakker, J.W., Meyer, J.-J.Ch., Olderog, E.-R.: Infinite streams and finite observations in the semantics of uniform concurrency. In: Proceedings 12th ICALP (W. Brauer, ed.), LNCS 194, pp. 149-157. Berlin-Heidelberg-New York: Springer 1985

5. de Bakker, J.W., Meyer, J.-J.Ch., Olderog, E.-R.: Infinite streams and finite observations in the semantics of uniform concurrency. Report CS-R 8512, Centre for Mathematics and Computer Science, 1985 (full version of [4], to appear in Theor. Comput. Sci.)

6. de Bakker, J.W., Meyer, J.-J.Ch., Olderog, E.-R., Zucker, J.I.: Transition systems, infinitary languages and the semantics of uniform concurrency. In: Proceedings 17th ACM STOC, pp. 252-262. ACM-Publications: Providence, R.I. 1985

7. de Bakker, J.W., Meyer, J.-J.Ch., Olderog, E.-R., Zucker, J.I.: Transition systems, metric spaces and ready sets in the semantics of uniform concurrency. Report CS-R 8601, Centre for Mathematics and Computer Science 1986 (full version of [6])

8. de Bakker, J.W., Zucker, J.I.: Processes and the denotational semantics of concurrency. Inf. Control 54, 70-120 (1982)

9. Bergstra, J.A., Klop, J.W.: Process algebra for synchronous communication. Inf. Control 60, $109-137$ (1984)

10. Broy, M.: Fixed point theory for communication and concurrency. IFIP TC 2 Working Conference 82, Garmisch-Partenkirchen (D. Bjørner, ed.). Amsterdam: North-Holland 1983

11. Broy, M.: A theory for nondeterminism, parallelism, communication and concurrency. Theor. Comput. Sci. 45, 1-62 (1986)

12. Dugundji, J.: Topology. Rockleigh, N.J.: Allen and Bacon 1966

13. Engelking, R.: General topology. Polish Scientific Publishers 1977

14. Hahn, H.: Reelle Funktionen. New York: Chelsea 1948

15. Hennessy, M., Plotkin, G.D.: Full abstraction for a simple parallel programming language. In: Proceedings 8th MFCS (J. Becvar, ed.), LNCS 74, pp. 108-120. Berlin-Heidelberg-New York: Springer 1979

16. Joshi, K.D.: Introduction to General Topology. New Delhi: Wiley Eastern 1983

17. Kuiper, R.: An operational semantics for bounded nondeterminism equivalent to a denotational one. IFIP TC2-MC Symp. on Algorithmic Languages (J.W. de Bakker, J.C. van Vliet, eds.), pp. 373-398. Amsterdam: North-Holland 1981 
18. Meyer, J.-J.Ch.: Programming calculi based on fixed point transformations: semantics and applications. Dissertation, Free University of Amsterdam, 1985

19. Meyer, J.-J.Ch.: Merging regular processes by means of fixed point theory. Theor. Comput. Sci. 45, 193-260 (1986)

20. Meyer, J.-J.Ch., de Vink, E.P.: Applications of compactness in the Smyth power domain of streams. Report IR-110, Free University, Amsterdam, 1986; extended abstract in Proceedings TAPSOFT/ CAAP'87 (H. Ehrig, R. Kowalski, G. Levi, U. Montanari, eds.), Pisa, LNCS 249, pp. 241-255. Berlin-Heidelberg-New York-Tokyo: Springer 1987

21. Nivat, M.: Infinite words, infinite trees, infinite computations, Foundations of Computer Science III.2. Math. Centre Tracts 109, 3-52 (1979)

22. Olderog, E.-R., Hoare, C.A.R.: Specification-oriented semantics for communicating processes. Acta Inf. 23, 9-66 (1986)

23. Plotkin, G.D.: A structural approach to operational semantics, Report DAIMI FN-19, Comp. Sci. Dept., Aarhus Univ. 1981

24. Rounds, W.C.: On the relationship between Scott domains, synchronization trees and metric spaces. Report Univ. of Michigan CRL-TR-25-83, 1983

Received November 20, 1986/May 18, 1987 